\title{
Conundrum: intervention for unprotected left main bifurcation coronary lesions
}

\author{
Shigeru Fukuzawa
}

Published online: 10 November 2010

(C) Japanese Association of Cardiovascular Intervention and Therapeutics 2010

Percutaneous coronary intervention (PCI) of the unprotected left main (LM) artery is currently not recommended as a routine procedure based on the history of inferior outcomes of LM percutaneous transluminal coronary angioplasty and bare metal stenting. Instead, surgical revascularization coronary artery bypass grafting (CABG) is considered to be the gold standard. There is renewed interest in LM-PCI because of improved outcomes of PCI utilizing drug eluting stents (DES) in multiple randomized trials. Several single-center non-randomized registries [1-3] have evaluated the role of DES for LM-PCI. Data suggest a low mortality and target vessel failure of ostial LM or mid-shaft lesions in contrast to bifurcation lesions, which frequently require complex dual stenting techniques. Actually, the treatment of lesions at LM bifurcations remains a technical challenge. Interventions at these sites are associated not only with a high risk of acute temporal deterioration of coronary flow but definitive loss of one of the distal large branches may also occur.

The recently reported SYNTAX trial [4] utilized several important design features which made these results the most relevant information available to guide the management of patients with advanced coronary artery disease (CAD). SYNTAX was a large prospective randomized controlled trial (RCT) for patients with three-vessel or LM (or combined) CAD. Its clinical importance is enhanced by its multicenter design and the enrollment of "all comers".

The online version of the article referred to in this editorial can be found under doi:10.1007/s12928-010-0039-7.

S. Fukuzawa $(\square)$

Division of Cardiology, Heart and Vascular Institute,

Funabashi Municipal Medical Center, 1-21-1 Kanasugi,

Funabashi, Chiba 273-8588, Japan

e-mail: sf-fuku@qa2.so-net.ne.jp
Patients eligible for randomization were those in whom a consensus was reached by an interdisciplinary team of interventional cardiologists and cardiothoracic surgeons that equivalent revascularization could be achieved with either therapeutic option. Subjects were prospectively stratified by diabetic status and the presence or absence of LM disease. In LM substudy, major adverse cardiac and cerebrovascular event (MACCE) rates at 1 year were similar for CABG and PCI (13.7 vs. $15.8 \%, P=0.44)$. At 1 year, stroke was significantly higher in the CABG arm (2.7 vs. $0.3 \%, P=0.009$ ), whereas repeat revascularization was significantly higher in the PCI arm (6.5 vs. $11.8 \%$, $P=0.02)$; there was no observed difference between groups for other end points. As this result, PCI to the LM disease to a morbid state became Class IIB from Class III in the guideline. However, it is short-circuited to conclude that only PCI can treat all the patients with LM disease. In the important point that should be noted with SYNTAX, there are 1077 examples (25\%) who are judged to be an adjustment of CABG of all examples. In contrast, it is only 198 examples that were deemed suitable only for PCI. It is only $59 \%$ that was randomized after all, and $35 \%$ of the case of the whole is assumed that the risk of PCI is high and registered in $\mathrm{CABG}$ registry. Going forward, insights into the selection of patients with LM or multivessel disease who can be optimally managed with PCI was provided by the SYNTAX score. The SYNTAX score was designed to reflect the complexity of coronary disease, based primarily on anatomic characteristics present on the diagnostic angiogram, with higher scores indicating more complex disease. For analysis purposes, the authors divided patients into one of three groups with respect to their SYNTAX score (low, medium and high). Among patients randomized to PCI, patients in the highest SYNTAX score tertile experienced significantly higher rates of MACCE at 
1 year compared to patients in the lowest tertile (23.4 vs. $13.6 \%, p=0.002)$.

On the other hand, too many interventional strategies for LM bifurcations have been proposed to address these adverse anatomic scenarios. This profusion of unsettled technique simply reflects our failure to provide clear answer to unmet needs. Indeed, full metal coverage of the origin of three segments stemming from the bifurcation is sometimes required to ensure satisfactory geometric results. However, this aggressive approach often leads to important additional problems, namely coronary segments with a double metal layer or with significant stent malposition. Accordingly, the pendulum is swinging back to simple solutions that, whenever feasible, are currently being widely advocated, taking into consideration the efficacy demonstrated by the use of DES in this setting. Furthermore, the pragmatism of accepting suboptimal angiographic result at the ostium of a jailed side branch is gaining popularity considering that most alternative therapeutic options are not very attractive.

In this issue of the Journal, Kato et al. report the short term acceptable angiographic results of the moderate side branch stenosis of LM bifurcation treated with single sirolimus-eluting stents (SES). This study is important because it extends our knowledge about the prevalence of no progression within 1 year of moderate stenosis in jailed LM side branch. However, to accept their conclusions, some consideration is needed. One is to have to understand the bias to the case's selection. The bias is large also even in a large-scale registry of SYNTAX as previously mentioned. Of the 3075 patients included in the trial, and 1275 were deemed suitable for only one treatment option, and of these, 198 were deemed suitable only for PCI. Therefore, there might be danger that a suitable case for the single stent implantation was chosen by priority in the present study. Moreover, how many cases were there CABG's having been deemed suitable in the LM bifurcation cases? It is suspected that the situation that the case where the residual stenosis of the side branch is $50 \%$ or less occupies half the number is developed because of this bias. Second, it is necessary to note that the end-point of the present study is not hard end-point named MACCE. That is, the end-point of the present study is only the surrogated endpoint which is described changing rate after 1 year of the residual stenosis of the daughter branches. The evidence whether for this surrogated end-point to influence the stent thrombosis and the restenosis in the future and to cause MACCE is not reported now. In addition, the authors described some trials for non-LM bifurcation coronary lesions instead of LM bifurcation in the discussion. In fact, during this 1 year, some publications [5-7] worthy of note regarding randomized analysis of stenting bifurcation coronary lesions have appeared. The consensus from the year's randomized trial data was that routine two vessel stenting did not improve either angiographic or clinical outcomes for most patients with coronary bifurcation lesions. Depending on one's view of the importance of periprocedural CK elevation, it could also be argued that routine dual vessel stenting did not involve a significant penalty either. Equally, kissing balloon inflations appeared neither beneficial nor harmful, but could justifiably be undertaken at the operator's discretion. However, this consensus is difficult as for the easy adjustment to LM bifurcation intervention. Previous IVUS studies [8, 9] reported that LM coronary artery bifurcation have demonstrated that atherosclerotic plaques are localized exclusively on the non-flow diving walls (opposite to carina) of the LAD and LCX. These prosperities make LM unique among all coronary arteries in the pathogenesis of atherosclerosis. These factors may explain the differential tissue characteristics between LM and non-LM bifurcations.

The present study focuses us on the importance of a simple stenting strategy for LM bifurcation. However, the present study was a little provocative when compared from the current evidence perspective.

Interventional strategy to LM bifurcations is still a conundrum, and more registry data and randomized trials are needed before unprotected LM-PCI can be routinely offered to patients as an alternative to CABG. Therefore, I would like to recommend that the single-center observation study of the PCI treatment to LM bifurcation should not be expanded under the present situation.

\section{References}

1. Palmerini T, Marzocchi A, Tamburino C, Sheiban I, Margheri M, Vecchi G, Sangiorgi G, Santarelli A, Bartorelli A, Briguori C, Vignali L, Di Pede F, Ramondo A, Inglese L, De Carlo M, Bolognese L, Benassi A, Palmieri C, Filippone V, Sangiorgi D, De Servi S. Two-year clinical outcome with drug-eluting stents versus bare-metal stents in a real-world registry of unprotected left main coronary artery stenosis from the Italian Society of Invasive Cardiology. Am J Cardiol. 2008;102(11):1463-8.

2. Palmerini T, Sangiorgi D, Marzocchi A, Tamburino C, Sheiban I, Margheri M, Vecchi G, Sangiorgi G, Ruffini M, Bartorelli AL, Briguori C, Vignali L, Di Pede F, Ramondo A, Inglese L, De Carlo M, Bolognese L, Benassi A, Palmieri C, Filippone V, Barlocco F, Lauria G, De Servi S. Ostial and midshaft lesions vs. bifurcation lesions in 1111 patients with unprotected left main coronary artery stenosis treated with drug-eluting stents: results of the survey from the Italian Society of Invasive Cardiology. Eur Heart J. 2009; 30(17):2087-94.

3. White AJ, Kedia G, Mirocha JM, Lee MS, Forrester JS, Morales WC, Dohad S, Kar S, Czer LS, Fontana GP, Trento A, Shah PK, Makkar RR. Comparison of coronary artery bypass surgery and percutaneous drug-eluting stent implantation for treatment of left main coronary artery stenosis. JACC Cardiovasc Interv. 2008;1(3):236-45.

4. Serruys PW, Morice MC, Kappetein AP, Colombo A, Holmes DR, Mack MJ, Ståhle E, Feldman TE, van den Brand M, Bass EJ, 
Van Dyck N, Leadley K, Dawkins KD, Mohr FW; SYNTAX Investigators. Percutaneous coronary intervention versus coronaryartery bypass grafting for severe coronary artery disease. N Engl J Med. 2009;360(10):961-72.

5. Erglis A, Kumsars I, Niemelä M, Kervinen K, Maeng M, Lassen JF, Gunnes P, Stavnes S, Jensen JS, Galløe A, Narbute I, Sondore D, Mäkikallio T, Ylitalo K, Christiansen EH, Ravkilde J, Steigen TK, Mannsverk J, Thayssen P, Hansen KN, Syvänne M, Helqvist S, Kjell N, Wiseth R, Aarøe J, Puhakka M, Thuesen L. Nordic PCI Study Group. Randomized comparison of coronary bifurcation stenting with the crush versus the culotte technique using sirolimus eluting stents: the Nordic stent technique study. Circ Cardiovasc Interv. 2009;2(1):27-34.

6. Colombo A, Bramucci E, Saccà S, Violini R, Lettieri C, Zanini R, Sheiban I, Paloscia L, Grube E, Schofer J, Bolognese L, Orlandi M, Niccoli G, Latib A, Airoldi F. Randomized study of the crush technique versus provisional side-branch stenting in true coronary bifurcations: the CACTUS (Coronary Bifurcations: Application of the Crushing Technique Using Sirolimus-Eluting Stents) Study. Circulation. 2009;119(1):71-8.

7. Ferenc M, Gick M, Kienzle RP, Bestehorn HP, Werner KD, Comberg T, Zhao M, Buettner HJ, Neumann FJ. Long-term outcome of percutaneous catheter intervention for de novo coronary bifurcation lesions with drug-eluting stents or bare-metal stents. Am Heart J. 2010;159(3):454-61.

8. Ding Z, Biggs T, Seed WA, Friedman MH. Influence of the geometry of the left main coronary artery bifurcation on the distribution of sudanophilia in the daughter vessels. Arterioscler Thromb Vasc Biol. 1997;17(7):1356-60.

9. Valgimigli M, Rodriguez-Granillo GA, Garcia-Garcia HM, Vaina S, De Jaegere P, De Feyter P, Serruys PW. Plaque composition in the left main stem mimics the distal but not the proximal tract of the left coronary artery: influence of clinical presentation, length of the left main trunk, lipid profile, and systemic levels of C-reactive protein. J Am Coll Cardiol. 2007;49(1):23-31. 